\title{
On the Growth and Polynomial Coefficients of Entire Series
}

\author{
Huzoor H. Khan ${ }^{1}$, Rifaqat Ali ${ }^{2}$ \\ ${ }^{1}$ Department of Mathematics, Aligarh Muslim University, Aligarh, India \\ ${ }^{2}$ Department of Applied Mathematics, Aligarh Muslim University, Aligarh, India \\ E-mail:huzoorkhan@yahoo.com,rifaqat.ali1@gmail.com \\ Received July 9, 2011; revised August 6, 2011; accepted August 13, 2011
}

\begin{abstract}
In this paper we have generalized some results of Rahman [1] by considering the maximum of $|f(z)|$ over a certain lemniscate instead of considering the maximum of $|f(z)|$, for $|z|=r$ and obtain the analogous results for the entire function $|f(z)|=\sum_{k=1}^{\infty} p_{k}(z)[q(z)]^{k-1}$ where $q(z)$ is a polynomial of degree $m$ and $p_{k}(z)$ is of degree $m-1$. Moreover, we have obtained some inequalities on the lover order, type and lower type in terms of polynomial coefficients.
\end{abstract}

Keywords: Lemniscate, Lower Order, Lower Type, Slowly Changing Function, Polynomial Coefficients and Entire Functions.

\section{Introduction}

Let

$$
f(z)=\sum_{n=0}^{\infty} a_{n} z^{n}
$$

be a nonconstant entire function and assume that $a_{n} \neq 0$ for $n=1,2,3, \cdots$ For classifying entire functions by their growth, the concept of order was introduced. If the order is a (finite) positive number, then the concept of type permits a subclassification. For the class of order $\rho$ $=0$ and $\rho=\infty$ no subclassification is possible. For example all entire functions that grow at least as fast as exp $(\exp (z))$ have to be kept in one class. For this reason, numerous attempts have been made to refine the concept of order and type. Boas [2] define the order $\rho(0 \leq \rho \leq \infty)$ and the type $T(0 \leq T \leq \infty)$ as follows:

$$
\begin{gathered}
\limsup _{r \rightarrow \infty} \frac{\log \log M(r, f)}{\log r}=\rho=\limsup _{n \rightarrow \infty} \frac{n \log n}{\log \left|a_{n}\right|^{-1}} \\
\limsup _{r \rightarrow \infty} \frac{\log M(r, f)}{r^{\rho}}=T=\limsup _{n \rightarrow \infty}\left(\frac{1}{e \rho} n\left|a_{n}\right|^{\rho / n}\right)
\end{gathered}
$$

where

$$
M(r, f)=\max _{|z|=r}|f(z)|,
$$

Rahman [1] studied the type by taking the function $r^{\rho}\left(\log _{1} r\right)^{a_{1}},\left(\log _{k} r\right)^{a_{k}}$ in place of $r^{\rho}$ and shown that

$$
\begin{aligned}
& \limsup _{r \rightarrow \infty} \frac{\log M(r, f)}{r^{\rho}\left(\log _{1} r\right)^{a_{1}}, \ldots . .\left(\log _{k} r\right)^{a_{k}}}=T(\rho) \\
& =\limsup _{n \rightarrow \infty}\left(\frac{\rho^{a_{1}-1}}{e} \frac{n\left|a_{n}\right|^{\rho / n}}{\left(\log _{1} n\right)^{a_{1}}, \ldots . .\left(\log _{k} n\right)^{a_{k}}}\right)
\end{aligned}
$$

In this paper, we show that instead of considering the maximum of $|f(z)|$, for $|z|=r$, we can consider the maximum of $f(z)$ over a certain lemniscate and obtained analogous results for entire function

$$
f(z)=\sum_{k=1}^{\infty} p_{k}(z)[q(z)]^{k-1},
$$

Walsh [3], Borwein [4], where $q(z)$ is a polynomial of degree $m$ and $p_{k}(z)$ is of degree $m-1$ and the equipotential curve $|q(z)|=R$ defines the lemniscate mentioned above, various authors such as Rice $([5,6])$, Juneja [7], Juneja and Kapoor [8], Kumar [9], Kumar and Kaur [10] studied the growth of above entire function but non of them studied the analogous results of (1.3). Therefore we have obtained lower order, type and lower type in terms of polynomial coefficients. 
Rice [5] has extended the results (1.1) and (1.2) for the $\quad$ lemniscate $\Gamma_{R} .:|q(z)|=R$, i.e.,

$$
\begin{gathered}
\limsup _{R \rightarrow \infty} \frac{\log \log M\left(\Gamma_{R}, f\right)}{\log R}=\rho / m=\limsup _{n \rightarrow \infty} \frac{n \log n}{\log \left[\left\|p_{n}(z)\right\|_{\Gamma a}\right]^{-1}}, \\
\limsup _{R \rightarrow \infty} \frac{\log M\left(\Gamma_{R}, f\right)}{R^{\rho / m}}=T=\limsup _{n \rightarrow \infty} \frac{m}{e \rho} n\left(\left\|p_{n}(z)\right\|_{\Gamma a}\right)^{\rho / m n},
\end{gathered}
$$

where

$$
M\left(\Gamma_{R}, f\right)=\left|f(z) \|_{\Gamma_{R}}=\max _{z \in \Gamma_{R}}\right| f(z),
$$

$\Gamma_{R}$ is the boundary of the lemniscate.

Analogous to (1.4) the lower order $\lambda$ if $f(z)$ can be defined as

$$
\frac{\lambda}{m}=\liminf _{R \rightarrow \infty} \frac{\log \log M\left(\Gamma_{R, f}\right)}{\log R}
$$

\section{Definitions and Auxiliary Results}

Definition 2.1. Slowly changing function $\phi(r)$ is defined as:

1) $\phi(r)$ is positive, continuous and tends to $\infty$ as $r \rightarrow \infty$,

2) $\lim _{r \rightarrow \infty} \frac{\varphi(k r)}{\varphi(r)}=1$, for every fixed $k>0$,

3) $\frac{\varphi\left[\left(\frac{x}{\varphi(x)}\right)^{1 / \rho}\right]}{\varphi(x)} \rightarrow \varphi(\rho) \neq 0$, bounded as $x \rightarrow \infty$.

Now we prove

Lemma 2.1. $f(z)=\sum_{k=1}^{\infty} p_{k}(z)[q(z)]^{k-1}$ is an entire function of order $\rho>0$ and type $T_{\phi}$ with respect to order $\rho$, if and only if,

$$
\limsup _{R \rightarrow \infty} \frac{\log M\left(\Gamma_{R}, f\right)}{R^{\rho / m} \varphi\left(R^{1 / m}\right)}=T_{\varphi} .
$$

Proof. Set $S=R^{I / m}(1+O(1))$, so that from the estimate Rice [5], $\left\|\Gamma_{R}\right\|=2 \pi R^{1 / m}(1+O(1))$ as $R \rightarrow \infty$ for $z \in \Gamma_{R},|z|=S$, we have

$$
\limsup _{R \rightarrow \infty} \frac{\log M\left(\Gamma_{R}, f\right)}{R^{\rho / m} \varphi\left(R^{1 / m}\right)}=\limsup _{S \rightarrow \infty}\left[\frac{\log M(S, f)}{\left(S^{\rho}(1+O(1))^{\rho} \varphi(S(1+O(1))\right.}\right]=\underset{S \rightarrow \infty}{\limsup } \frac{\log M(S, f)}{S^{\rho} \varphi(S)}=T_{\varphi} .
$$

Lemma 2.2. $f(z)=\sum_{k=1}^{\infty} p_{k}(z)[q(z)]^{k-1}$

is an entire function of order $\rho>0$ and lower type $T_{\phi}$, if and only if

$$
\liminf _{R \rightarrow \infty} \frac{\log M\left(\Gamma_{R}, f\right)}{R^{\rho / m} \varphi\left(R^{1 / m}\right)}=t_{\varphi} .
$$

Proof. Proof can be done in a similar manner as Lemma 2.1.

\section{Main Results}

First we prove the inequality for lower order $\lambda$ in terms of polynomial coefficients.

Theorem 3.1. Let $\alpha$ be fixed and

$$
f(z)=\sum_{k=1}^{\infty} p_{k}(z)[q(z)]^{k-1}
$$

be an entire function of order $\rho>0$ and lower order $\lambda$,

$$
\log M\left(\Gamma_{R}, f\right) \geq \log \left(\frac{2 \pi R^{k}}{\left\|\Gamma_{R}\right\|\|Q(z)\|_{\Gamma_{R}}}\left\|p_{k}(z)\right\|_{\Gamma_{\alpha}}\right)>-\frac{k \log k}{\gamma-\varepsilon}+k \log R-\log \frac{\left\|\Gamma_{R}\right\|}{2 \pi}\|Q(z)\|_{\Gamma_{R}}
$$


Choose $R=e k^{1 /(\gamma-\varepsilon)}$, since $\alpha<R$, for sufficiently large $k$, we obtain

$$
\log M\left(\Gamma_{R}, f\right)>k \log \left(R k^{-1}\right)^{-1 /(\gamma-\varepsilon)}-\log \frac{\left\|\Gamma_{R}\right\|}{2 \pi}\|Q(z)\|_{\Gamma_{R}}=k-\log \frac{\left\|\Gamma_{R}\right\|}{2 \pi}\|Q(z)\|_{\Gamma_{R}}
$$

or

$$
\log M\left(\Gamma_{R}, f\right)>(R / e)^{\gamma-\varepsilon}-\log \frac{\left\|\Gamma_{R}\right\|}{2 \pi}\|Q(z)\|_{\Gamma_{R}}
$$

or

$$
\log \log M\left(\Gamma_{R}, f\right)>(\gamma-\varepsilon) \log (R / e)-\log [1-(e / R)]^{(\gamma-\varepsilon)} \log \frac{\left\|\Gamma_{R}\right\|}{2 \pi}\|Q(z)\|_{\Gamma_{R}}
$$

In view of a result of Rice [5]

$$
\frac{\left\|\Gamma_{R}\right\|}{2 \pi}=R^{1 / m}(1+O(1))
$$

we get

$$
\frac{\log \log M\left(\Gamma_{R}, f\right)}{\log R}>(\gamma-\varepsilon)+O(1) .
$$

Proceeding to limit as $R \rightarrow \infty$, we get

$$
\limsup _{r \rightarrow \infty} \frac{\log M(r, f)}{r^{\rho} \phi(r)}=T_{\varphi}=\frac{m}{e \rho \varphi(\rho)} \limsup _{k \rightarrow \infty} \frac{k}{\phi(k)}\left(\left\|p_{k}(z)\right\|_{\Gamma_{\alpha}}\right)^{\rho / m k}
$$

Proof. Let

$$
\beta=\limsup _{k \rightarrow \infty} \frac{k}{\phi(k)}\left(\left\|p_{k}(z)\right\|_{\Gamma_{\alpha}}\right)^{\rho / m k} .
$$

Then for a given $\varepsilon>0$, we get

$$
\left\|\rho_{k}(z)\right\|_{\Gamma \alpha} \geq\left(\frac{(\beta-\varepsilon)}{k} \phi(k)\right)^{m k / \rho} .
$$

For an infinite sequence of values of $k$, so that from (3.1), we have

$$
M\left(\Gamma_{R}, f\right) \geq \frac{2 \pi\left[R^{\rho / m} \frac{(\beta-\varepsilon)}{k} \phi(k)\right]^{m k / \rho}}{\left\|\Gamma_{R}\right\|\|Q(z)\|_{\Gamma_{R}}}
$$

or

Choosing a sequence of values of $R$ such that

$$
R^{\rho / m}=\frac{k e}{(\beta-\varepsilon)} \phi(k) .
$$

For these $R$ the right hand side of (3.3) attains its maximum value, that is,

$$
M\left(\Gamma_{R}, f\right) \geq \frac{2 \pi(e)^{m k / \rho}}{\left\|\Gamma_{R}\right\|\|Q(z)\|_{\Gamma_{R}}},
$$

$$
\log M\left(\Gamma_{R}, f\right) \geq \frac{m k}{\rho}+\log 2 \pi-\log \left[\left\|\Gamma_{R}\right\|\|Q(z)\|_{\Gamma_{R}}\right]
$$

or

$$
\frac{\log M\left(\Gamma_{R}, f\right)}{R^{\rho / m} \varphi\left(R^{1 / m}\right)} \geq \frac{m}{\rho} \frac{k}{R^{\rho / m} \phi\left(R^{1 / m}\right)}+O(1)=\frac{m}{\rho} \frac{(\beta-\varepsilon)}{e} \frac{\phi(k)}{\phi\left[\left(\frac{k e}{\phi(k)(\beta-\varepsilon}\right)^{1 / \rho}\right]}+O(1)
$$

Applying the limits as $R \rightarrow \infty$, we get

$$
T_{\phi} \geq \frac{m}{\rho} \frac{(\beta-\varepsilon)}{e} \frac{1}{\varphi(\rho)}
$$

$\frac{k}{\phi(k)}\left(\left\|p_{k}(z)\right\|\right)^{\rho / m k} \leq \beta+\varepsilon$, for sufficiently large $k$.

In order to prove reverse inequality, from (3.2) we have

Now following the same manner as in the proof of (3.4), we get 


$$
\mathrm{T}_{\phi} \leq \frac{m(\beta+\varepsilon)}{e \rho \varphi(\rho)}
$$

Since $\varepsilon$ is arbitrary, combining (3.4) and (3.6) we get

$$
\mathrm{T}_{\varphi}=\frac{m \beta}{e \rho \varphi(\rho)}
$$

Now we have to prove that $T_{\phi}$ is a type of $f(\mathrm{z})$ with respect to order $\rho$, when

$$
\mathrm{T}_{\varphi}=\frac{m \beta}{e \rho \varphi(\rho)}
$$

In fact we can assume that the inequality (3.5) holds for all $k$ as we can always add a polynomial to $f(z)$ without afficting its order. Thus

$$
|f(z)|=\left|\sum_{k=1}^{\infty} p_{k}(z)[q(z)]^{k-1}\right| \leq \sum_{k=1}^{\infty}\left|P_{k}(k)\right| R^{k-1} \leq \sum_{k=1}^{\infty}\left\|p_{k}(z)\right\|_{\Gamma_{\alpha}} R^{k+m-2} \leq R^{m-2} \sum_{K=1}^{\infty}\left(\frac{(\beta+\varepsilon)}{k} \phi(k)\right)^{m k / p} R^{k}
$$

For $k=k_{1}$, given by $k_{1}=(\beta+\varepsilon) \phi\left(k_{1}\right) R^{\rho / m} e^{O(1)-1}$, we find that

$$
\begin{gathered}
\left(\frac{(\beta+\varepsilon) \varphi(k)}{k}\right)^{m k / \rho} \\
\text { Now choosing } K_{1} \text { such that } K_{1} \leq k_{1}<K_{1}+1 \text {. Then } \\
R^{m-2} \sum_{k=1}^{\infty}\left(\frac{(\beta+\varepsilon)}{k} \phi(k)\right)^{m k / \rho} R^{k}=R^{m-2} \sum_{k=1}^{K_{1}}\left(\frac{(\beta+\varepsilon)}{k} \phi(k)\right)^{m k / \rho} R^{k}+\sum_{k=K_{1}+1}^{\infty}\left(\frac{(\beta+\varepsilon)}{k} \phi(k)\right)^{m k / \rho} R^{k} \\
\leq K_{1} R^{m-2} \exp \left\{[1-O(1)] \frac{m}{\rho}(\beta+\varepsilon) \varphi\left(k_{1}\right) R^{\rho / m} e^{O(1)-1}+R^{m-2} N^{*}\right\}
\end{gathered}
$$

where $N^{*}$ is the sum of the convergent series $\sum_{k=K_{1}+1}^{\infty}\left(\frac{(\beta+\varepsilon)}{k} \phi(k)\right)^{m k / \rho} R^{k}$.

So

$$
\begin{gathered}
M\left(\Gamma_{\mathrm{R}}, f\right)=\|f(z)\|_{\Gamma_{R}} \leq K_{1} R^{m-2} \exp \left\{[1-O(1)] \frac{m}{\rho}(\beta+\varepsilon) \phi\left(k_{1}\right) R^{\rho / m} e^{O(1)-1} \cdot(1+O(1))\right\} \\
\leq(\beta+\varepsilon) \phi\left(k_{1}\right) R^{\rho / m} e^{O(1)-1} R^{m-2} \exp \left\{[1-O(1)] \frac{m}{\rho}(\beta+\varepsilon) \phi\left(k_{1}\right) R^{\rho / m} e^{O(1)-1} \cdot(1+O(1))\right\}
\end{gathered}
$$

which gives

$$
\frac{\log M\left(\Gamma_{R,} f\right)}{R^{\rho / m} \phi\left(R^{1 / m}\right)} \leq \frac{(1-O(1)) \frac{m}{\rho}(\beta+\varepsilon) \varphi\left(k_{1}\right) R^{\rho / m} e^{O(1)-1}}{R^{\rho / m} \varphi\left(R^{1 / m}\right)}+O(1)=(1-O(1)) \frac{m}{\rho} \frac{(\beta+\varepsilon)}{e} e^{O(1)} \frac{\phi\left(k_{1}\right)}{\phi\left[\left(\frac{k_{1} e}{\phi\left(k_{1}\right)(\beta+\varepsilon)}\right)^{1 / \rho}\right]}+O(1) .
$$

In view of Lemma 2.1 and condition (3) of Definition 2.1 , we get

$$
T_{\varphi} \leq \frac{m}{\rho} \frac{(\beta+\varepsilon)}{e} \frac{1}{\varphi(\rho)} .
$$

Similarly we can prove

$$
T_{\varphi} \geq \frac{m}{\rho} \frac{(\beta-\varepsilon)}{e} \frac{1}{\varphi(\rho)},
$$

by taking

$$
\frac{k}{\phi(k)}\left(\left\|p_{k}(z)\right\|_{\Gamma_{\alpha}}\right)^{\rho / m k} \geq \beta-\varepsilon,
$$

for an infinite sequence if values of $k \rightarrow \infty$ and choosing a corresponding sequence of values of $R$ such that

$$
R=\left(\frac{e}{(\beta-\varepsilon)} \frac{k}{\varphi(k)}\right)^{m / \rho}
$$

with (3.1). Hence the proof is complete.

Theorem 3.3. Let $\alpha$ be fixed. If

$$
f(z)=\sum_{k=1}^{\infty} p_{k}(z)[q(z)]^{k-1}
$$

is an entire function of order $\rho>0$ and lower type $t_{\varphi}$, where

$$
t_{\varphi}=\liminf _{r \rightarrow \infty} \frac{\log M(r, f)}{r^{\rho} \varphi(r)},
$$


Then

$$
\frac{m}{e \rho \varphi(\rho)} \liminf _{k \rightarrow \infty} \frac{k}{\phi(k)}\left(\left\|p_{k}(z)\right\|_{\Gamma_{\alpha}}\right)^{\rho / m k} \leq t_{\phi}
$$

Proof. The proof of this theorem follows on the lines of the necessary part of Theorem 3.2 , by writing

$$
\beta^{\prime}=\liminf _{k \rightarrow \infty} \frac{k}{\phi(k)}\left(\left\|p_{k}(z)\right\|_{\Gamma_{\alpha}}\right)^{\rho / m k}
$$

and for a given $\varepsilon>0$,

$$
\frac{k}{\phi(k)}\left(\left\|p_{k}(z)\right\|_{\Gamma_{\alpha}}\right)^{\rho / m k}>\left(\beta^{\prime}-\varepsilon\right) \text { for } k \geq K(\varepsilon),
$$

so that we may choose a sequence of values of $R$ satisfying

$$
R^{\rho / m}=\frac{k}{\varphi(k)} \frac{e}{\left(\beta^{\prime}-\varepsilon\right)} .
$$

Now using the relation (3.1) and Lemma 2.2, we get the required result. Hence the proof is complete.

Remark 3.1. Theorem 3.2 is the generalization of the result (1.3) by Rahman [1].

Remark 3.2. Rahman's theorem [1], if

$$
\frac{T(\rho)}{k}=\liminf _{r \rightarrow \infty} \frac{\log M(r, f)}{r^{\rho}\left(\log _{1} r\right)^{\alpha_{1}}, \ldots .\left(\log _{k} r\right)^{\alpha_{k}}}
$$

then

$$
T(\rho) \geq \liminf _{n \rightarrow \infty}\left(\frac{\rho^{\alpha_{1}-1}}{e} \frac{n}{\left(\log _{1} n\right)^{\alpha_{1}}, \ldots .\left(\log _{k} n\right)^{\alpha_{k}}}\left|a_{n}\right|^{\rho / n}\right)
$$

is a special case of Theorem 3.3, if we take $\phi(r)=\left(\log _{1} r\right)^{a_{1}}, \ldots\left(\log _{k} r\right)^{a_{k}}$ and consider the circle $|\mathrm{z}|=$ $r$, instead if the lemniscate $|q(z)|=R$.

\section{References}

[1] Q. I. Rahman, "On the Coefficients of an Entire Series of Finite Order," Math Student, Vol. 25, 1957, pp. 113-121.

[2] R. P. Boas, "Entire Functions," Academic Press, New York, 1954, pp. 9-11.

[3] J. L. Walsh, "Interpolation and Approximation," American Mathematical Society, Colloquim Publications, Providence, Vol. 20, 1960, p. 56.

[4] P. Borwein, "The Arc Length of the Lemniscate $\{|p(z)|=$ $1\}$," Proceedings of the AMS-American Mathematical Society, Vol. 123, 1995, pp. 797-799.

[5] J. R. Rice, "A Characterization of Entire Functions in Terms of Degree of Convergence," Bulletin of the American Mathematical Society, Vol. 76, 1970, p. 129. doi:10.1090/S0002-9904-1970-12396-5

[6] J. R. Rice, "The Degree of Convergence for Entire Functions," Duke Mathematical Journal, Vol. 38, No. 3, 1971, pp. 429-440. doi:10.1215/S0012-7094-71-03852-X

[7] O. P. Juneja, "On the Coefficients of Entire Series," Journal of Mathematical Analysis, Vol. 24, 1971, pp. 395-401.

[8] O. P. Juneja and G. P. Kapoor, "Polynomial Coefficients of Entire Series," Yokohama Mathematical Journal, Vol. 22, 1974, pp. 125-133.

[9] D. Kumar, "Approximation Error and Generalized Orders of an Entire Function," Tamsui Oxford Journal of Mathematical Sciences, Vol. 25, No. 2, 2009, pp. 225235.

[10] D. Kumar and H. Kaur, " $L^{p}$-Approximation Error and Generalized Growth Parameters of Analytic Functions in Coratheodory Domains," International Journal of Mathematical Analysis, Vol. 3, No. 30, 2009, pp. 1461-1472. 